\title{
Design of a Unified Data with Business Rules Storage Model for OLTP and OLAP Systems
}

\author{
Stephen Opoku-Anokye and Yinshan Tang \\ Informatics Research Centre, Henley Business School, University of Reading, United Kingdom
}

This paper reviews the literature concerning the practice of using Online Analytical Processing (OLAP) systems to recall information stored by Online Transactional Processing (OLTP) systems. Such a review provides a basis for discussion on the need for the information that are recalled through OLAP systems to maintain the contexts of transactions with the data captured by the respective OLTP system. The paper observes an industry trend involving the use of OLTP systems to process information into data, which are then stored in databases without the business rules that were used to process information and data stored in OLTP databases without associated business rules. This includes the necessitation of a practice, whereby sets of business rules are used to extract, cleanse, transform and load data from disparate OLTP systems into OLAP databases to support the requirements for complex reporting and analytics. These sets of business rules are usually not the same as business rules used to capture data in particular OLTP systems. The paper argues that differences between the business rules used to interpret these same data sets risk gaps in semantics between information captured by OLTP systems and information recalled through OLAP systems. The literature concerning the modeling of business transaction information as facts with context as part of the modelling of information systems was reviewed to identify design trends that are contributing to the design quality of OLTP and OLAP systems. The paper then argues that the quality of OLTP and OLAP systems design has a critical dependency on the capture of facts with associated context, encoding facts with contexts into data with business rules, storage and sourcing of data with business rules, decoding data with business rules into the facts with the context and recall of facts with associated contexts. The paper proposes UBIRQ, a design model to aid the co-design of data with business rules storage for OLTP and OLAP purposes. The proposed design model provides an opportunity for the implementation and use of multi-purpose databases and business rules stores for OLTP and OLAP systems. Such implementations would enable the use of OLTP systems to record and store data with executions of business rules, which will allow for the use of OLTP and OLAP systems to query data with business rules used to capture the data, thereby ensuring that information recalled via OLAP systems preserves the contexts of transactions as per the data captured by the respective OLTP system.
Keywords: OLTP systems design, OLAP systems design, facts with contexts modelling, data with business rules modelling

\section{Introduction}

Requirements for Online Analytical Processing (OLAP) are usually not considered during the design of systems to satisfy Online Transactional Processing (OLTP) requirements. Traditionally, OLTP requirements gathering exercises are focused mainly on transaction processing. The need for analytical processing becomes apparent only when OLTP systems are in use and begin to generate large volumes and complex varieties of data at high frequencies. It is at this point that OLAP systems are designed specifically to extract, transform and load data from OLTP systems into OLAP databases to support analytical requirements. The lack of consideration for the transaction processing requirements, together with analytical processing requirements, presents many issues, challenges and problems when OLAP systems are used to interpret data stored in OLTP databases. The facts and contexts of information captured during transaction processing are usually stored and interpreted by computers in the form of data and business rules (i.e. rule flow and logic). Traditionally, the design of systems to support OLTP and OLAP requirements usually followed a two tier architecture design pattern (i.e. an application tier that embeds the business rules used for transactions or analytical processing and data 
tier used for storage or sourcing of data). Sometimes, the components that make up these two tiers could be separated into several layers. An example is described by Fowler (2003) as three principal layers (i.e. presentation, domain and data source) and by Microsoft (2009) as three software design layers (i.e. presentation, business and data). Another example is the five software designed layers, which is described by Sun Microsystems (2005) as composed of the client, presentation, business, services and data. Irrespective of how many layers the design of an OLTP system or OLAP system may be divided into, business rules are often stored as an integral part of the core of OLTP and OLAP systems. Data, however, are most often stored in databases that are separate to the core of the OLTP system or OLAP system. These are contributing to the necessity of practice whereby data are extracted from different OLTP systems into databases dedicated for use by OLAP systems, in order to support the organizational need for complex reporting and analytics. The data in these databases exclude original business rules used by particular OLTP systems to process data. The use of these databases by OLAP systems for analytical processing is, therefore, achieved with only transactional data. Extraction of data with business rules used to process the data, however, becomes very difficult, and, if at all possible, their use to maintain the context of information captured during transaction processing may not be possible. Business rules are usually recreated within OLAP systems, resulting in a possible difference between the semantics of information captured by OLTP systems and information recalled via OLAP systems.

Design of OLTP or OLAP databases focuses primarily on efficient and effective ways of implementing systems for either transactional or analytical use. The primary focus of OLTP systems is on capturing, processing and storing of data as and when business transactions occur. Database systems that support OLTP requirements are often designed to improve the efficiency of processing transactions, collecting information about processed transactions and storing capture data to a persistent storage. Limitations in computer technology constrain the design of persistent data storage for OLTP systems. For example, duplications of data that are stored in the persistent storage are reduced in order to increase the efficiency of process- ing business transaction. This is to reduce the number of times that an OLTP system will have to save data entities or objects into a persistent store (i.e. in the case of an OLTP system, the ideal number of times is once). To help achieve these objectives, an array of information systems modelling methods and techniques are adopted during OLTP systems design. The use of most of these modelling methods and techniques to design OLTP systems renders inefficient the use of OLAP systems to access the data stored in persistent storage, that were designed for use by OLTP systems. Especially the requirements to support organizational needs for complex reporting, querying and analytics. In some cases, OLTP databases may even be unsuitable for use by OLAP systems, given the structure of the data stored by a particular OLTP System. Database systems that support OLAP requirements are often designed to improve the efficiency of processing, analytical data, retrieving information about processed transactions, consolidating and aggregating data stored in multiple data repositories. OLAP systems have a primary focus to extract, transform and load data after business transactions have occurred for the purposes of reporting, querying and analytics. During the design of OLAP systems, this process is popularly known as extract, transform and load (ETL). The ETL process involves the extraction of data from several OLTP persistent data stores, cleansing, transforming and loading data into a consolidated persistent data store that is mainly for OLAP use. The extraction, cleansing and transformation of data involves the use of business rules that are elicited for the sole purpose of ETL. Such business rules usually have no direct relationship with the business rules used to capture data into respective OLTP systems.

The current common industry practice involves the use of OLTP systems to process information into data, which are then stored in databases without the business rules that were used to process information. The use of these data without business rules, excludes critical details about the facts of information captured. These exclusions include information about what, how, when, where and why a particular piece of information was processed during business operations and decision making cycles. Business operational contexts of these captured facts exist within the data stored and business rules 
that are used to process the information. Thus, data stored in OLTP databases without associated business rules, may not have the same context as original information captured. In order to maintain business operational contexts of information captured, OLTP systems need to capture facts with contexts, encode these facts and contexts into data using business rules and then store the data with associated executions of business rules. In this paper, we review the modeling of business transaction information as facts with context. We propose an approach to the co-design of data with business rules storage for OLTP and OLAP purposes. The proposed design model provides an opportunity for the implementation and use of multi-purpose databases, and business rules stores for OLTP and OLAP systems. The integration of data storage with business rules executions would provide support for the use of OLAP systems to query data with the business rules that were used to capture such data. These ensure that the facts of information recalled through OLAP systems maintain the business operational context of information captured by particular OLTP systems.

\section{Modeling of Facts with Business Operational Context}

The information required to support business operations and decision making include signs used to represent such information (syntax), meaning of these signs (semantics) and purposeful use of these signs (pragmatics). Signs are usually in the form of text, numbers, symbols, "words, image, sounds, gestures, and objects" (Chandler 2007). These are the raw data that represent the syntax of the information processed by OLTP systems during business transaction processing and also information processed by OLAP systems during complex reporting, querying and analytics. Davenport and Pruzak (2000) define data as "a set of discrete, objective facts about events," while Newell et al. (2009) define data as "a record of signs and observations collected from different sources." Information, however, is described by Newell et al. (2009) as data that are "presented in a particular way in relation to a particular context of action." Contexts of actions in an OLTP system are, as per the processing of business transactions. Semantics of data captured by OLTP systems during processing of business transaction are embedded in relevant facts and associated contexts. Professor Henderson quoted in (Parsons 1949) refers to a Fact as "empirically verifiable statement about phenomena." In the case of OLTP systems, transactions are phenomena that occur during business operations and decision making cycles. The 'contexts of actions' for these phenomena include:

- Life cycle and value chain of the products that are produced, as the results of business decisions and operations

- Organizations involved in producing these products and their organizational structure, processes, management and strategy

- Operating environments and relationships with all stakeholders, internal or external

Dey (2001) defines the context as "any information that can be used to characterize the situation of an entity." The contexts of actions that are captured by OLTP systems during the processing of transactions encodes details about the circumstances and conditions within which transactions occur; and thus influences the meaning and purposeful use of data stored in OLTP databases (see Figure 1).

Information system modeling is a system analysis and design technique used to elicit, analyze and specify business and solution requirements, and also to design and document OLTP and OLAP systems. Halpin and Bloesch (1999) describe information systems modeling from two perspectives, i.e. (1) data perspective and (2) process and behavioral perspective. OLTP and OLAP systems represent implementations of computer-supported information systems within and across organizations. Their designs thus include modeling from the data point of view and the point of view of process and behavior. Modelling from the data perspective focuses designers' attention to what information is to be encoded (Soames 1989). Information is encoded into data when an OLTP system is used to process business transactions. OLAP systems, however, are used to decode the data stored in databases optimized for OLTP use into information for the purposes of business reporting, querying and analysis. From a data modelling perspective, the transaction can be seen as what 


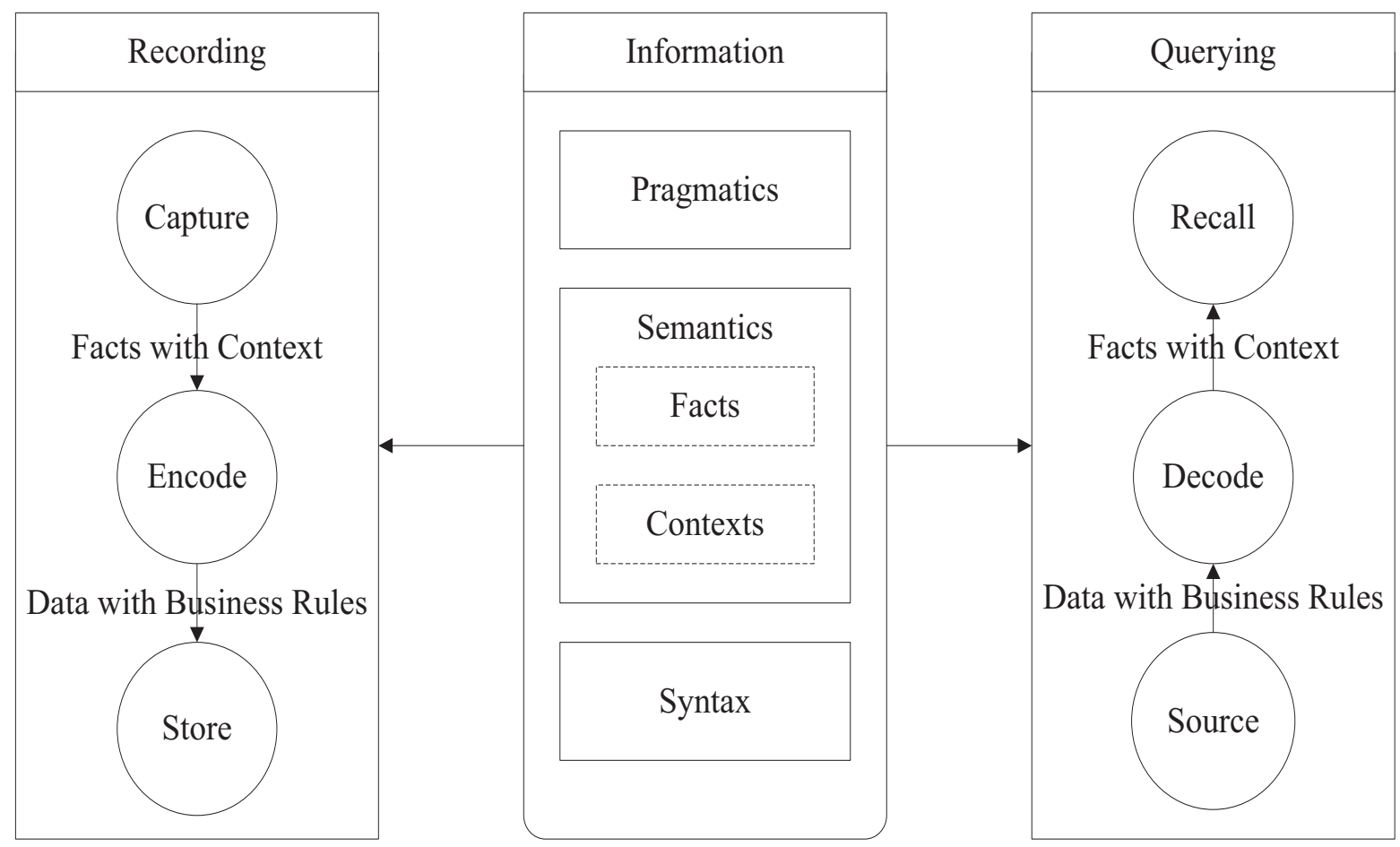

Figure 1. Information recording and querying with OLTP and OLAP systems.

Connolly and Begg (2005) described as "an action or series of actions, carried out by a single user or application program, which access or change the content of the database." OLTP systems can be described as application programs that carry out actions or support end-users to carry out a series of actions, which access or change the contents of a database or databases. OLAP systems, on the other hand, can be described as application programs that are used to access and extract data from disparate operational databases into an analytical database or databases and then change the content of the analytical database for the purpose of reporting, querying and analytics. As explained by Davenport and Pruzak (2000), "data becomes information when its creator add meaning" and, also, data is translated into information "by adding value in various ways", including contextualization, categorization, calculation, correction and condensation. OLTP systems use business rules to create and store data during the processing of transactions. These business rules are thus required by OLAP systems in order to add meaning to the data created and stored by OLTP systems. The modeling of process and behavior, focus designers' attention on executions of business rules used to process transactions and on how these business rules are associ- ated with the data created and stored by processing of such transactions. A model for execution of business rules during transaction processing provides abstractions that represent the realities of situations and conditions within which transactions are processed. This includes modelling of business rules that are used to process, record or query information. Business rules are 'assertions that constrain patterns' of process executions during business operations and decisions making cycles. They include policies and standards that govern business operations (Morabito et al. 1999, Bajec and Krisper 2005). So, the business rules model can be used to represent abstractions, structuring, formalization and transformations (Oberweis 1996) of business processes, practices and behaviors.

Beynon-Davies (2002) describes the process of modeling an information system as construction of sign-systems for reasons of communication, representation and abstraction of conditions in the real world. A model in this sense is an approximation of realities in business operations and decision making environments. Use of modeling, therefore, enables system analysts and designers to explore and improve their understanding of organizations, associated systems, business processes, practices and the wider business environments (Stair 
and Reynolds 2011). The industry committee commonly known as ANSI/SPARC published in 1978 documents that described components of a three-level architecture for information systems, which included external, conceptual and internal models. The external model is a model for application programs that are used to access and change contents of a database. External models are used to communicate, represent and provide an abstraction of how OLTP and OLAP system users view and use information. External modelling of OLTP and OLAP systems can thus be referred to as the modelling of application programs. This includes designing for information processing (i.e. encoding information into data and decoding data into information), integration and presentation. Internal models for OLTP and OLAP systems provide abstract views of where and how information is stored or sourced. It is a model for the contents and structure of a database or databases. Although not often considered as part of the internal model, the contents and structure of business rules stores are part of the internal modelling. Internal modelling of OLTP or OLAP systems includes logical and physical data modelling and also logical and physical modelling of business rules. The physical distribution of internal models can be different from the understanding of their logical distributions (Moss and Atre 2003). Conceptual models provide for the mapping of external models to internal models, as such; they are used as representations of system specifications to communicate system designs to developers and information systems users alike. This includes detailed specifications of requirements for OLTP and OLAP systems to record or query information. Conceptual models are independent of any technology used to implement external and internal models.

We argue that the quality of OLTP and OLAP systems design has a critical dependency on the following:

- Capture of facts with associated context

- Encoding facts with contexts into data with business rules

- Storage and sourcing of data with business rules

- Decoding data with business rules into the facts in the context

- Recall of facts with associated contexts
These should be modelled at the conceptual level and are best designed with particular attention to facts captured during transaction processing, contexts within which these facts are captured, business rules used for processing transactions and associated data. Therefore, it is important for systems designers to consider not just the data, but rather data with the execution of business rules during external, conceptual and internal modelling of OLTP or OLAP systems. Co-design of data models and business rules execution models ensure the linkage of facts with the contexts of transactions to storage of data and execution of business rules. Parsons (1949) clarified that a fact is not in itself a phenomenon, "but a proposition about one or more phenomena." It is thus possible for a particular fact to be associated with one or more business operational contexts. Likewise, data stored in OLTP databases may be associated with one or many executions of business rules. The execution of business rules is another category of records that are fundamental to the use of OLTP systems to support the processing of business transactions. Storage of business rule executions includes information about when they were triggered and how they affect the data stored (Bajec and Krisper 2005). Storage of business rules and associated executions with data will provide capabilities for the use of OLAP systems to study interactions among business rules (Rai and Anantaram 2004), and also the interactions between business rules and associated data. Such study can be used in business intelligence, which aims to provide information to support evidence based decision making.

\section{Unified Information Recording and Querying Model}

We propose that the design of OLTP and OLAP systems be based on a unified business information recording and querying (UBIRQ) and the principles of computer-supported information recording (i.e. capture, encode and store) and querying (i.e. source, decode and recall), as described in Figure 1. The design approach (see Figure 2) is composed of three models, i.e. Online Transactional Processing (OLTP), 


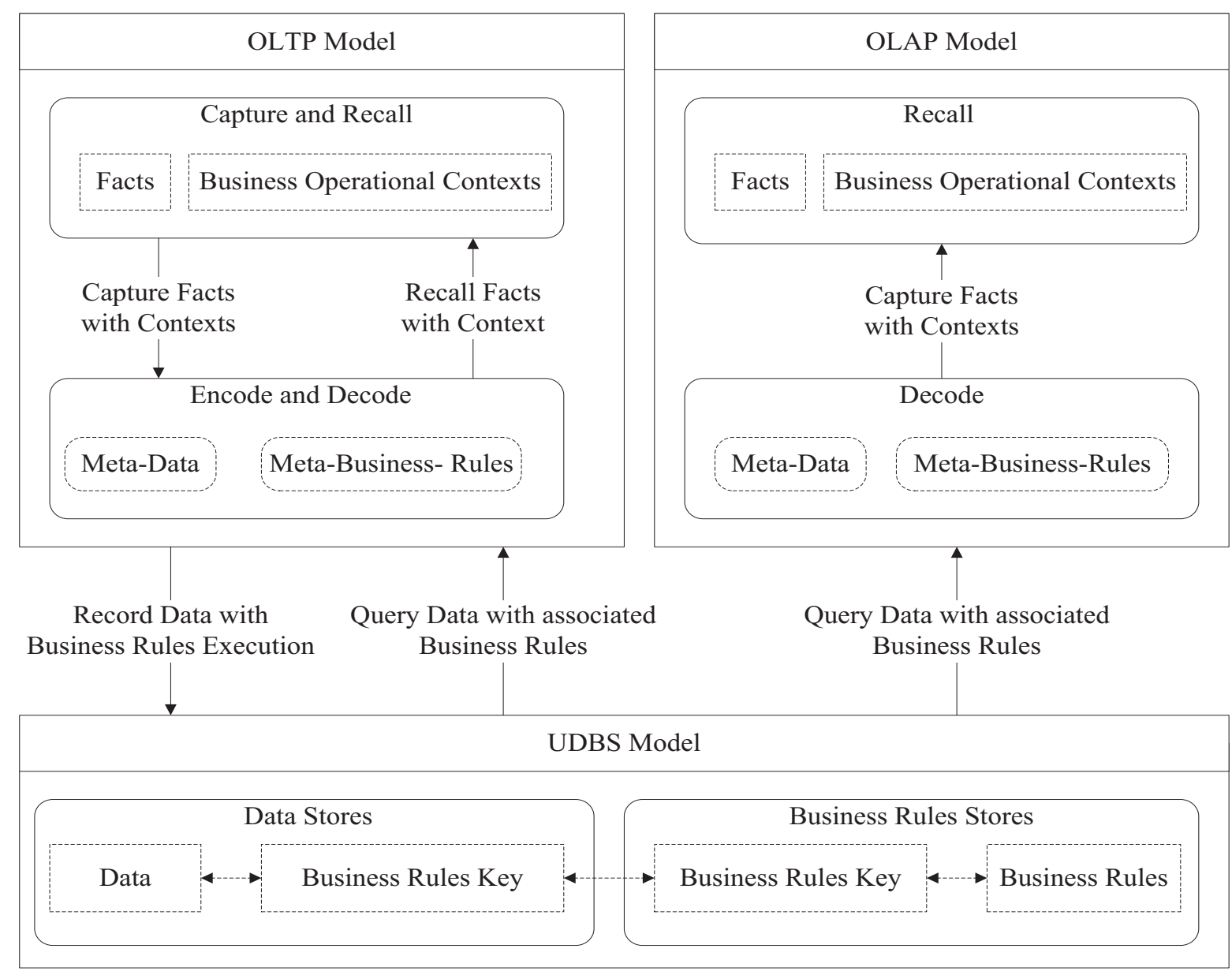

Figure 2. Unified data with business rules storage model for OLTP and OLAP systems.

Online Analytical Processing (OLAP) and unified data with business rules store (UDBS). Design of OLTP models considers capture, display and storage of data with business rules to support processing of business transactions. OLAP models design considers reporting, querying and analysis of data with business rules that were used to process the data. The UDBS model can be used to help co-design data with business rules storage to support OLTP and OLAP requirements. UDBS allows data that are linked to business rules used to produce such data to be designed for storage in databases that are separate from the core of OLTP or OLAP systems. Just as data, business rules are also designed to be stored in business rules stores that are separate from the core of the respective OLTP or OLAP systems. This does not only make possible the extraction of data with business rules used to create the data, but also makes possible their use to preserve the context of information captured during processing of business trans- actions. The use of OLAP systems to process data captured by OLTP systems is, therefore, achieved with, not just the data captured, but in the context within which transactions were processed. Linkage of data with business rules during systems design focuses the attention of designers on the use of business rules by OLTP systems, to capture and encode facts with contexts into data for storage, as well as, on the use of business rules to source, decode and recall data into the facts with contexts that are presented to end-users.

Computer-supported OLTP and OLAP systems are not capable of understanding facts with context in the same way as humans do, hence, the need to encode the facts with contexts into data with business rules for a computer to process. Also, the need to decode the data with business rules into the facts with contexts for the people in the organization to aid their understanding. OLTP systems are modeled to encode facts with transactional context into data, using the 
business rules stored in a unified business rules repository for use by both OLTP and OLAP systems. Data with associated executions of any of these business rules are then recorded into a database. This enables OLTP and OLAP systems to source data with business rules from databases and business rules stores and then to decode them into facts with contexts. Encoding facts with contexts into data with business rules and decoding data with business rules into the facts with contexts are achieved by the use of meta-data and meta-business-rules. Metadata contains information about data stored within respective databases, while meta-business-rules contain information about business rules that are stored within respective business rules stores. Data stored in databases are linked to the associated business rules in particular business rules stores by having a business rule executions keys for each data record link to business rules keys in respective business rules stores. Two types of meta-data and meta-business-rules are required during OLTP systems design that is based on the UBIRQ model. These are

1. meta-data and meta-business-rules that describe how information about business transactions are captured, encoded and stored in data with business rules stores

2. meta-data and meta-business-rules that describe how data with business rules are sourced from their respective storage for decoding and display as facts with contexts

These meta-business-rules could be designed using a locking and unlocking mechanism with associated business rules key that is stored together with the data in the unified data store. During the design of OLAP systems, meta-data and meta-business-rules that describe how data with business rules are sourced from the unified storage for decoding and presentation as facts with contexts are consolidated. This ensures that the information contained in reports, dashboards, scorecards and query results from OLAP systems can be interpreted within the relevant business operational context. The codesign and unified approach to the storage of data with business rules enable business operational context of information captured by OLTP systems to be preserved when such information are being recalled, either through OLTP or OLAP systems (see Figure 2).
Advances in technologies in recent years have meant that varieties of technologies are now available for supporting implementations of OLTP or OLAP systems design using the UBIRQ approach. These include technologies and techniques that allow the following:

- Use of in-memory database architectures and faster disk-drive architectures to provide fast read-write to and from database management systems (DBMS).

- Implementation of business rules stores using business rules management systems (BRMS) together with storage and retrieval of business rules executions.

- OLTP and OLAP systems to use meta-data and meta-business-rules for recording and querying information to and from DBMS and BRMS.

The above mentioned technologies and techniques make it possible for the implementation of OLTP and OLAP systems that make use of databases and business rules stores designed on the basis of a physical implementation of UDBS model. These do also make possible physical implementations of multiple and disparate OLTP and OLAP systems based on the logical designs of OLTP or OLAP models.

\section{Discussions}

Today, organizations have information that is typically stored in databases optimized for transactional (i.e. OLTP systems) or analytical (OLAP systems) use. OLTP systems are designed to process large volumes of business transactions at high frequencies. As such OLTP databases are designed to capture and store large amounts of data, as and when generated during processing of business transaction. Design efforts for OLTP systems focus on how to provide support for more efficient and productive operations (Duan and Xu 2012). The main issues considered during OLTP design are processing, display and storage of data. Capture of business rules and logic are not considered as important. Databases for OLTP use are designed primarily to support processing of concurrent transactions, usually in their hundreds, thousands or millions, depending on the size of the organization or enterprise. Data is usually extracted from OLTP systems into OLAP systems 
for transformation into information, purposefully to support the business need for complex reporting, querying and analytics. Often, the data extracted and transformed are loaded into databases dedicated for the purpose of generating management reports and analytical use. These databases include data warehouses, data marts, data cubes and many other data storage systems. It can be argued from an OLAP design perspective that information stored about transactions by OLTP systems are usually formed by two parts, i.e. business rules (including rule flow and logic used to process the transaction) and data created pre, during and post processing of transactions. Data stored within databases designed for OLTP systems can also be argued to be the footprints of business transactions processed by the OLTP system. Such data represent what 'action or series of actions' have been carried out. Making data created by OLTP systems available and accessible for use by OLAP systems, provides support for a process that was described by (Golfarelli et al. 2004) as "turning data into information and then into knowledge". Such data or information or knowledge embeds that facts with contexts that allow decision makers across organizations to answer questions relating to what has happened in connection with the processing of business transactions. Business rules, which include logic and rule flows used to process transactions, can be said to represent abstract views of processing steps for business transactions. These business rules are representations of decisions that are, or should be, taken by OLTP systems on whether or not an 'action or series of actions' are carried out during the processing of particular business transactions. Therefore, making available and accessible the business rules that are used by OLTP systems to process business transactions, and in formats that are usable to OLAP systems would provide support for the use of OLAP systems to decode data into information, using business rules that were used by the respective OLTP system to encode the data. Such information would not only allow decision makers' answer questions relating to what has happened in connection with business transactions, but also allow them to answer questions relating to how particular business transactions are processed. In some cases, these may also make available information concerning why particular business transactions are processed.
Data modelling exercises are undertaken during the design of OLTP systems to develop abstract models that allow physical implementation of OLTP systems to process, collect, store and retrieve data for the purposes of supporting business transactions processing. During the design of OLAP systems, data modelling exercises are undertaken to develop abstract models allowing physical implementation of OLAP systems to source data and present facts with context. Such presentation of facts with contexts enables stakeholders explore, analyze, query and synthesis of available data into useful information to support operational, tactical and strategic decision making. It is important for designers who design data models for OLTP systems to understand organizational requirements for OLAP, to allow them to make design decisions that include:

1. Access strategies for data that would be captured and stored by the OLTP system,

2. Information about data stored (i.e. metadata) and why and how the data were processed (i.e. meta-business-rules)

3. Presentation of information to end-users for use to satisfy analytical requirements

Designers need to question repeatedly whether or not data that are captured and stored by OLTP system and associated business rules used for processing the data would support OLAP requirements. Also, how up to date these data with associated business rules need to be in relations to processing of transactions, in order to satisfy requirements for OLAP and complex reporting. Repeated questions of applicability of captured information for OLAP purposes during the design of OLTP systems provide answers that reshape design strategies for data access, meta-data, meta-business-rules, data retrieval and facts with contexts presentation. For example, the design of unified data with business rules storage model, for use by both OLTP and OLAP systems do offer a better solution to the business requirements for complex reporting and OLAP systems that dictate for the availability of real time information. Design of OLAP systems, therefore, need to include identification of OLTP data and business rules sources that contain information required to satisfy analytical need. Designers of data models for OLAP systems need to give due consideration to 
the appropriateness of OLTP data and business rules sources and reuse of OLTP data models for presenting facts with contexts to business users.

Models and technologies that are currently used to implement OLTP systems make a typical OLTP database and business rules store very complex and often unsuitable for complex reporting and analytics. Entity Relationship (ER) modelling of data is a technique traditionally used by designers' of data models for OLTP systems to help analyze OLTP data requirements and design their logical and physical data structures. Dimensional data model design is a technique predominately used for $\log$ ical design of data structures for OLAP systems. Kimball (1997) described it as "logical design technique that seeks to present the data in a standard, intuitive framework that allows for high-performance access." The focus for dimensional data model design is placed on the provision of improved capabilities for complex reporting and also for business users to analyze and visualize data. The two primary assumptions that are usually made during dimensional data model design include the notions that:

- Captured data about transactions are records of business operational activities and results of information processed by respective OLTP systems,

- OLTP data models are organized abstractions of business operational data.

These emphasize the use of data models as natural and better method for understanding, communicating and managing business data. It does, however, exclude business rules used for processing transactions and analytical information. Models for these business rules are an important part of the information processed and as such are required to add context to the data captured and stored during transaction processing. Dimensional data model design technique is commonly used in industry to design data structures for data marts, data warehouses and historical operational data stores, to make them dimensionally aware data sources. Another current use of the dimensional data model design technique is a meta-data model design technique known as 'OLAP over Relational,' which is used to make OLTP data sources, that are not dimensionally aware data sources, appear to an OLAP system, as if they were dimensionally aware data sources.
Business rules govern organizational processes and activities and are used to process, by OLTP systems, databases and OLAP systems to process data. Despite their importance, business rules are not captured, stored and treated with importance similar to that of data. It is common practice for business rules to be hard coded into the fabrics of OLTP systems, by OLAP systems and their respective databases. This makes it very difficult to capture and store business rules used in OLTP systems for OLAP purposes. Even if OLAP developers go through the tedious process of extracting these business rules (i.e. Logic and rule flow) from their respective OLTPs systems, their use to satisfy the organizational need for complex reporting and analytics will be very limited. The capture of data into databases by OLTP systems without associated execution of business rules governing the processing of such data exclude the context through which the data were generated. Data without context in which it was generated allows for the data to be misinterpreted or interpreted in the wrong context. Consequently, the information obtained through any OLAP system based on the data without the business rules used will be context deficient in terms of the circumstances in which the information was captured. Without the contexts by which the data was captured, processed, encoded and stored, attempts to transform the data into meaningful information using OLAP may result in various degrees of semantic gaps between information as existed and processed by the respective OLTP system and the information obtained through reporting, exploration and analysis using OLAP systems. The origins of gaps in semantics between OLTP and OLAP can be summarized into three main categories, which are:

1. The operative terms of reference and measurement units used by different functions of an organization may be varied

2. Multiple stakeholder groups use OLAP systems to transform data captured and stored within OLTP systems into information to support decision making at different levels of management and information granularity.

3. The use of OLAP systems to interpret data captured and stored within databases designed for OLTP systems, without associated business rules used to generate the data. 
Semantics gaps based on categories (1) and (2) could be resolved within the business domain by implementing common standards for operational terms of reference and measurement units. Semantics gaps based on category (3) could be resolved when the following two conditions are met:

- OLTP system captures, stores and treats business rules with importance similar to that of the data

- Information obtained through OLAP systems are sourced from the data in association with the business rules used to process such data

The UBIRQ model proposed in this paper provides a solution to category (3) based semantic gaps if the model is used as a blueprint for designing of data with business rules storage for OLTP and OLAP purposes. It provides logical unification of business rules stored, their association with and use by OLTP and OLAP systems, and having each executed business rule key linked to each data record stored within a logically unified business data store. UBIRQ enables data stored within OLTP systems to be associated with the business rules to process the data and thereby allows for the use of OLAP systems to interpret data captured and stored within OLTP databases with associated business rules used to generate the data.

\section{Conclusion}

Business operational information that is captured and stored by OLTP systems represents footprints of business transactions and activities. The practice of extracting data from disparate OLTP systems for transformation and loading into OLAP databases are meant to satisfy business requirements to provide information for the purposes of supporting organizational decision making. OLAP, that is based on the data without business rules used to capture data, enables decision makers to answer questions relating to what has happened during business operations and decision making cycles. Business rules, which include rule flow and logic, contain details of decisions taken by OLTP systems and people during transaction processing, including situations and conditions under which the data were or are being generated. Thus, OLAP that is based on the data with business rules used to capture data enables decision makers to answer questions relating to the circumstances and conditions within which transactions were or are being processed. Storage of business rules, together with data as part of records kept by OLTP systems, will ensure facts with context recalled using OLAP systems based on data with business rules used to capture the data. UBIRQ model enables co-design of OLTP and OLAP databases as well as business rules stores. Thereby insured subsequent implementation allows for a unified approach to recording and querying of information related to business transactions. Ensuring facts recalled from OLAP systems will have the same contexts of actions as the facts captured and stored by OLTP systems. This helps to preserve business operational context from information capture during transaction processing, irrespective of whether it is being queried by OLTP or OLAP systems.

\section{References}

[1] M. BAJEC, M. KRISPER, A methodology and tool support for managing business rules in organisations. Information Systems, 30(6) (2005), 423-443.

[2] P. BEYNON-DAVIES, Information systems: An introduction to informatics in organisations. Palgrave, 2002.

[3] D. Chandler, Semiotics: The basics Abingdon. UK: Routledge, 2007.

[4] T. M. Connolly, C. E. BegG, Database systems: A practical approach to design, implementation, and management, 4th Edition. London, UK: AddisonWesley, 2005.

[5] T. H. DAVENPORT, L. PRUZAK, Working knowledge: How organizations manage what they know. Harvard Business Press, 2000.

[6] A. K. DEY, Understanding and using context. Personal and ubiquitous computing, 5(1) (2001), 4-7.

[7] L. DuAn, L. XU, Business intelligence for enterprise systems: A survey. Industrial Informatics, IEEE Transactions on PP, 99 (2012), 1-1.

[8] M. FowLER, Patterns of enterprise application architecture. Addison-Wesley Professional, 2003.

[9] M. Golfarelli, S. Rizzi, I. Cella, Beyond data warehousing: What's next in business intelligence? Proceedings of the 7th ACM International Workshop on Data warehousing and OLAP, (2004) pp. 1-6. Washington, DC, USA: ACM. 
[10] T. Halpin, A. Bloesch, Data modeling in uml and orm: A comparison. Journal of Database Management (JDM), 10(4) (1999), 4-13.

[11] R. KIMBALL, A dimensional modeling manifesto. $D B M S, 10(9)$ (1997), 58-70.

[12] MicrosofT, Microsoft application architecture guide, 2nd Edition. 2009.

[13] J. Morabito, I. SACK, A. Bhate, Organization modeling: Innovative architectures for the 21st century. Prentice Hall PTR, 1999.

[14] L. T. Moss, S. ATRE, Business intelligence roadmap: The complete project lifecycle for decision-support applications. Addison-Wesley Professional, 2003.

[15] S. Newell, M. Robertson, H. Scarbrough, J. SWAN, Managing knowledge work and innovation. Palgrave Macmillan, 2009.

[16] A. OBERWEIS, An integrated approach for the specification of processes and related complex structured objects in business applications. Decision support systems, 17(1) (1996), 31-53.

[17] T. PARSONS, The structure of social action. Free Press, New York, 1949.

[18] V. K. RaI, C. ANANTARAM, Structuring business rules interactions. Electronic Commerce Research and Applications, 3(1) (2004), 54-73.

[19] S. SOAMES, Semantics and semantic competence. Philosophical Perspectives, 3 (1989), 575-596.

[20] R. M. StAIR, G. W. REYNOLDS, Principles of information systems: Cengage Learning, 2011. Sun Microsystems, I., 2005. Sun java enterprise system 2005q1 technical overview.

Received: October, 2013 Revised: April, 2014 Accepted: July, 2014
Contact addresses:

Stephen Opoku-Anokye Informatics Research Centre

Henley Business School

University of Reading Whiteknights

Reading, RG6 6UD

United Kingdom

e-mail: s.opoku-anokye@pgr.ac.uk

Yinshan Tang Informatics Research Centre Henley Business School

University of Reading Whiteknights

Reading, RG6 6UD

United Kingdom

e-mail: y.tang@henley.ac.uk

STEPHEN OPOKU-ANOKYE is a part-time Ph.D. student who is researching the integration of business intelligence into information systems design at the Informatics Research Centre, Henley Business School, University of Reading. He is a dedicated team player and leader with over 8 years of experience in design, analysis, development, and implementation of information systems (IS). He has a successful history of leading business intelligence (BI) projects and architecting IS and BI solutions. He has worked on all phases of the IS development lifecycle, from gathering requirements to testing, implementation, and support. Prior to the inception of his Ph.D. research, he had worked as an IS Analyst/Programmer. At the inception of research, he worked as an IS Architect/BI Developer. In the course of the research, he worked as Business Rules Systems Designer/Developer, Principal Lead BI Developer/Consultant and currently he is the Manager of an enterprise-wide BI development team.

DR. YINSHAN TANG is an associate professor of Business Informatics, a Vice Dean of Henley Business School, University of Reading. He received his first degree in Biology from Hebei Agricultural University in 1982, a PhD in Parasitology and Medical Entomology from Imperial College London in 1994 and an MSc in Computer Science from Staffordshire University in 1999. He led and conducted large research projects on epidemiology of tropical diseases, chemical ecology, e-government, enterprise architecture, e-policing and sustainable carbon strategies. He acquired experience in industry work as a senior manager for over 6 years before joining University of Reading in 2004 to establish the Informatics Research Centre. His research interest lies in interdisciplinary research areas such as cross cultural management and decision making, knowledge management, business and technology infrastructure, information sharing, bioinformatics and sustainable strategies etc. 
